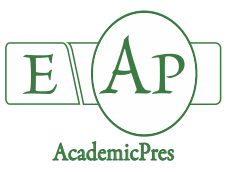

Koskosidis A et al. (2020)

Notulae Botanicae Horti Agrobotanici Cluj-Napoca 48(1):294-304

DOI:10.15835/nbha48111799

Research Article

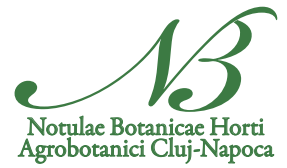

\title{
Effect of PEG-induced drought stress on germination of ten chickpea (Cicer arietinum L.) genotypes
}

\author{
Avraam KOSKOSIDIS ${ }^{1}$, Ebrahim KHAH $^{1}$, \\ Athanasios MAVROMATIS ${ }^{2}$, Ourania PAVLI ${ }^{1}$, \\ Dimitrios N. VLACHOSTERGIOS ${ }^{3 *}$
}

\author{
${ }^{1}$ University of Thessaly, School of Agricultural Science, Laboratory of Genetics and Plant Breeding, Fytokou Str., 38446 Volos, \\ Greece;makisk47@gmail.com;ekhah@uth.gr; raniapavli@yahoo.gr \\ ${ }^{2}$ Aristotle University of Thessaloniki, Laboratory of Genetics and Plant Breeding, School of Agriculture, 54124 Thessaloniki, \\ Greece; amavromat@agro.auth.gr \\ ${ }^{3}$ Hellenic Agricultural Organization Demeter, Institute of Industrial and Forage Crops, 41335 Larissa, \\ Greece; vlachostergios@gmail.com (*correspondingauthor)
}

\begin{abstract}
Chickpea (Cicer arietinum L.) is one of the most important legume crops worldwide, with its importance mainly relying on its high protein content. Chickpea productivity is strictly threatened by abiotic stresses, of which drought exerts the most crucial role in terms of growth inhibition and yield losses encountered. Given that germination is a critical stage that is negatively affected by drought, the aim of this study was to estimate the genotypic variability among ten chickpea genotypes and to determine the seed germination and seedling growth ability under drought stress conditions. Seeds were subjected to water stress by polyethylene glycol (PEG-6000) at five stress levels (0, 5, 10, 20, 30 and 50\% PEG). Germination percentage, seed water content, seed water absorbance, root and shoot development and seedling vigour index were evaluated. The analyses revealed significant genetic variability in relation to genotypic performance under drought stress. Drought significantly affected germination as well as all other associated traits, with the effects of stress being analogous to the stress level applied. Findings point to the fact that seedling vigour index is a suitable selection criterion for drought tolerance as it allowed the classification of genotypes as tolerant, moderately tolerant, moderately susceptible and susceptible. Taken together, the commercial varieties 'Thiva', 'Keryneia' and 'Gavdos' as well as the landrace 'Lemnos' showed an increased drought tolerance at high stress level, indicating their possible exploitation as valuable genetic material for breeding programs or for commercial use.
\end{abstract}

Keywords: chickpea; drought; germination; tolerance; water stress

Abbreviations: PEG: polyethylene glycol; WU: Seed water absorbance; WC: Seed water content; SVI: seedling vigor index 


\section{Introduction}

Chickpea (Cicer arietinum L.) is an important pulse crop cultivated in over 50 countries (Jumrani and Bhatia, 2014). Chickpea is a grain legume of great dietary value mainly due to its high protein content (Venn and Mann, 2004; Duc et al., 2014; Arnoldi et al., 2015). Further, its ability for nitrogen fixation contributes significantly to the reduction of fertilizer inputs (Rubiales and Mikic, 2015). Chickpea is classified as a lowwater-demanding crop with satisfactory adaptation in dry areas, with crop establishment however being largely depended on seed germination potential under low water availability (Arjenaki et al., 2011).

During the last 5000 years, limited water availability is considered as the main factor hampering crop production worldwide (Ceccarelli et al., 2010). Drought stress adversely affects crops throughout their lifecycle and causes yield losses, the extent of which varies significantly depending on the duration and intensity of the stress, the genetic background and the developmental stage. Moderate to severe drought reduces plant biomass, grain yield and related yield components of legumes (Siddique et al., 2001), while estimates of yield losses due to terminal drought range from $35 \%$ to $50 \%$ (Sabaghpour, 2003). In chickpea, drought causes yield losses ranging from $30 \%$ to $60 \%$, depending on geographical region and length of crop season.

Considering the ongoing climate change as well as the gradual decline of available water resources, research efforts towards developing of drought tolerant germplasm is of paramount importance. Breeding for the trait is routinely based on evaluation of yield performance under water-deficit stress conditions in the field, a procedure which is rather slow and laborious due to the difficulties both in terms of obtaining homogeneous stress conditions and identifying drought tolerant genotypes (Shaheen and Hood-Nowotny, 2005). As an alternative, Richards (1978) underpinned germination phase as the most sensitive stage in chickpea lifecycle and proposed that selection at this phase may form a useful screening criterion for drought tolerance. Further, previous studies underline that seedling vigour index, which combines seed germination rate with morphological traits during early growth of seedlings, is a reliable index for screening drought tolerance at germination phase (Siahsar et al., 2010). In vitro screening constitutes an alternative selection method that is advantageous over the routine approaches as it is readily applicable and time-effective. To this purpose, stress is commonly conducted through the use of osmotic solutions, among which polyethylene glycol has proven a valuable means of inducing drought stress in the context of screening in vitro a large set of genotypes (Kulkarni and Deshpande, 2007). Polyethylene glycol is capable of simulating water stress through decreasing the osmotic potential without entrance into the plant cells (Rai et al., 2011). Previous studies on chickpeas (Kalefetoglu et al., 2009; Yucel et al., 2010) proved the efficiency of PEG to induce drought associated effects on both germination and seedling growth.

The objective of this experiment was to study the effect of PEG-induced drought stress in germination and other traits related to germination and post-germination growth. Specifically, the germination percentage, seed water uptake, seedling water content, root and shoot length and seedling vigour index were employed as parameters for estimating genetic variation among ten chickpea genotypes and for selecting drought tolerant genotypes to be employed for breeding purposes or for commercial use.

\section{Materials and Methods}

\section{Plant material}

The genetic material consisted of both local and foreign chickpea germplasm. The local material under study consisted of one landrace ('Lemnos'), two elite lines ('Sifnos', 'Line 9/14') and three commercial varieties ('Gavdos', 'Keryneia', 'Thiva'), developed by Hellenic Agricultural Organization-DEMETER/Inst. of Industrial and Forage Crops. The foreign germplasm referred to three elite lines ('CAT16-31', 'CAT16-27', 'CAT16-4'), originating from ICARDA, and one commercial variety, namely 'Macarena'. 


\section{Treatments and experimental design}

Drought stress was induced by PEG-6000 (M.W. 6000) at different stress levels: i) 0\% (control), ii) 5\%, iii) $10 \%$, iv) $20 \%$, v) $30 \%$ and vi) $50 \%$. According to Michell and Kaufmann (1972), the osmotic potential $\left(\psi_{s}\right)$ of aqueous solutions of polyethylene glycol 6000 (PEG-6000) is curvilinearly related to concentration, thus allowing for calculation of $\psi_{\text {s }}$, from known concentrations of PEG-6000 through an equation:

$$
\begin{gathered}
\psi s=-\left(1.18 \times 10^{2}\right) \times C-\left(1.18 \times 10^{-4}\right) \times C^{2}+\left(2.67 \times 10^{-4}\right) \times C \times \mathrm{T} \\
+\left(8.3910^{-7}\right) \times C^{2} \times \mathrm{T}
\end{gathered}
$$

where $\mathrm{C}$ is the concentration of PEG-6000 in $\mathrm{g} / \mathrm{kg} \mathrm{H}_{2} \mathrm{O}$ and $\mathrm{T}$ is the temperature in ${ }^{\circ} \mathrm{C}$ (Michell and Kaufmann, 1972).

Based on the abovementioned equation, the osmotic pressure of PEG solutions was estimated as follows: i) $0 \%$ (control), ii) $5 \%(-0.05 \mathrm{MPa})$, iii) $10 \%(-0.15 \mathrm{MPa})$, iv) $20 \%(-0.5 \mathrm{MPa})$, v) $30 \%(-1 \mathrm{MPa})$ and vi) $50 \%$ (2.7 $\mathrm{MPa})$.

Seeds were surface-sterilized for five minutes in $10 \%$ bleach solution and washed three times with sterile water. In order to avoid contamination, seeds were placed in plastic boxes with absorbent paper and treated with fungicide. Seeds were subsequently watered with $12 \mathrm{ml}$ of the appropriate PEG solution. Plastic boxes were placed under natural sunlight and room temperature (app. $25^{\circ} \mathrm{C}$ ). The experimental design used was a randomized complete block design with four replications for every genotype-stress level combination, each replication consisting of twenty seeds.

\section{Measurements}

Evaluation of drought tolerance was performed using the following parameters: germination percentage (\%) ( $1^{\text {st }}$ until $7^{\text {th }}$ day), seed water absorbance (WU) (\%) $\left(3^{\text {rd }}, 5^{\text {th }}\right.$ and $7^{\text {th }}$ day), seed water content (WC) (\%) $\left(10^{\text {th }}\right.$ and $15^{\text {th }}$ day) root and shoot length $(\mathrm{cm})\left(5^{\text {th }}, 7^{\text {th }}, 9^{\text {th }}, 11^{\text {th }}, 13^{\text {th }}\right.$ and $15^{\text {th }}$ day) and seedling vigor index (SVI) $\left(15^{\text {th }}\right.$ day). Seeds were considered germinated when the radicle had a length of at least $1 \mathrm{~mm}$. The estimation of abovementioned parameters was performed using the following formulas:

Seed water absorbance (WU) $(\%)=\frac{\mathrm{W} 2-\mathrm{W} 1}{\mathrm{~W} 2} \times 100$ (Mujeeb-ur-Rahman et al., 2008),

where $\mathrm{W}_{1}=$ initial seed weight and $\mathrm{W}_{2}=$ seed weight following water absorbance.

Seed water content $(\mathrm{WC})(\%)=\frac{\text { Fresh weight }- \text { Dry weight }}{\text { Fresh weight }} \times 100$ (Black and Pritchard, 2002)

Seedling vigor index $(\mathrm{SVI})=$ seedling length $(\mathrm{cm}) \times$ germination percentage (Abdul-Baki and Anderson, 1973), where seedling length = root leght + shootlegnth.

Further, correlations between germination and seed characteristics, namely 100 seed weight, WU, WC, shoot length, root length, were estimated.

\section{Statistical analysis}

Data were analyzed using ANOVA ( $\mathrm{p} \leq 0.05$ ), combining PEG concentrations and genotypes. Genotypes were compared within stress level at specific time intervals, as described above. Additionally, genotypes were compared across stress levels. Differences between means were compared by the least significant difference (LSD) test. All statistical analyses were performed using SPSS statistical software v. 20.

\section{Results}

Seed germination was significantly affected by drought stress while, at the same time, differing substantially among genotypes under study (Table 1). 
Table 1. Final germination (\%), as affected by genotype (G) and PEG concentration (C)

\begin{tabular}{|c|c|c|c|c|c|c|c|c|}
\hline \multirow{3}{*}{ Time } & \multirow{3}{*}{$\begin{array}{c}\text { Genotype } \\
\text { (G) }\end{array}$} & \multicolumn{7}{|c|}{ PEG Concentration (\%) (C) } \\
\hline & & \multirow{2}{*}{0} & \multirow{2}{*}{$\begin{array}{c}5 \\
(-0.05 \mathrm{MPa})\end{array}$} & \multirow{2}{*}{$\begin{array}{c}10 \\
(-0.15 \mathrm{MPa})\end{array}$} & \multirow{2}{*}{$\begin{array}{c}20 \\
(-0.5 \mathrm{MPa})\end{array}$} & \multirow{2}{*}{$\begin{array}{c}30 \\
(1 \mathrm{MPa})\end{array}$} & \multirow{2}{*}{$\begin{array}{c}50 \\
(-2.7 \mathrm{MPa})\end{array}$} & $(\%)$ \\
\hline & & & & & & & & Mean $(\mathrm{C})$ \\
\hline \multirow{11}{*}{$\begin{array}{l}7^{\text {th }} \\
\text { day }\end{array}$} & 'Gavdos' & 95 & 96 & 98 & 74 & 31 & 8 & $67 \mathrm{ab}$ \\
\hline & 'Keryneia' & 94 & 91 & 100 & 96 & 18 & 9 & $67.9 \mathrm{ab}$ \\
\hline & 'Macarena' & 96 & 94 & 89 & 35 & 0 & 0 & $52.3 \mathrm{~d}$ \\
\hline & 'CAT16-31' & 86 & 91 & 94 & 90 & 0 & 0 & $60.2 \mathrm{bcd}$ \\
\hline & 'CAT16-27’ & 93 & 96 & 48 & 0 & 28 & 0 & $44 \mathrm{e}$ \\
\hline & 'Thiva' & 99 & 100 & 99 & 99 & 30 & 0 & $71 \mathrm{a}$ \\
\hline & 'CAT16-4’ & 98 & 98 & 95 & 10 & 24 & 0 & $54 \mathrm{~cd}$ \\
\hline & 'Sifnos' & 98 & 95 & 88 & 85 & 0 & 0 & $60.8 \mathrm{bc}$ \\
\hline & 'Line 9/14' & 94 & 91 & 83 & 53 & 11 & 0 & $55.2 \mathrm{~cd}$ \\
\hline & 'Lemnos' & 95 & 88 & 79 & 76,30 & 79 & 3 & $70 \mathrm{a}$ \\
\hline & $\operatorname{Mean}(\mathrm{G})$ & $94.6 \mathrm{a}$ & $94 \mathrm{a}$ & $87 \mathrm{~b}$ & $61.8 c$ & $22 \mathrm{~d}$ & $2 \mathrm{e}$ & \\
\hline
\end{tabular}

Note: At each time interval (days), means followed by the same letter, within each factor, are not significantly different according to $\operatorname{LSD}(\mathrm{p} \leq 0.05)$.

At stress level, the germination percentage ranged from $94.6 \%$ to $2 \%$ in the controls and plants subjected to high stress level (50\% PEG), respectively. At low stress level (5\% PEG), no significant differences were noted, while higher stress levels $(10,20,30$ and 50\% PEG) resulted in substantial differences in germination percentage. Although certain differences were observed in the performance of genotypes at $10 \%$ PEG, most profound decrease in mean germination rate (germination rate across all genotypes at a certain PEG concentration) was recorded at transition from $20 \%$ to $30 \%$ PEG and was $39.8 \%$. Specifically, at 20\% PEG, 'Thiva', 'Keryneia' and 'CAT 16-31' showed a germination percentage above 90\%, while at 30\% PEG the entire set of genotypes presented considerably lower germination rates (ranging from 0-31\%), with the exception of landrace 'Lemnos' which retained a high germination rate (79\%). At high stress level (50\% PEG), germination was totally repressed in the majority of genotypes, with the exception of landrace 'Lemnos' and cultivars 'Gavdos' and 'Keryneia' that were capable of germination, presenting though low germination rates (3\%, 8\% and $9 \%$, respectively). Across stress levels, 'Thiva' and 'Lemnos' presented the highest values for mean germination percentage ( $71 \%$ and $70 \%$, respectively), followed by 'Keryneia' and 'Gavdos' (67.9\% and 67\%, respectively). In contrast, the most severe effect in germination was noted in cultivars 'Macarena' and 'CAT1627 , which showed mean germination rates of $52.3 \%$ and $44 \%$, respectively.

As expected, WC and WU increased over time in all genotypes as well as stress levels applied (Tables 2 and 3). At specific time intervals however, WC and WU showed a gradual decrease as PEG concentration increased. In relation to stress levels, the mean values of WC and WU did not differ between control and 5\% PEG treatment, whereas at all other stress levels significant differences were recorded. Across stress levels, 'Sifnos' and 'CAT16-31' presented the highest mean WU, while 'Gavdos' and 'Keryneia' showed the highest mean WC. At high stress level (50\% PEG), the best performing genotypes were 'CAT16-31' for WU and 'Line 9/14' and 'Keryneia' for WC. 
Table 2. Seedling water content (WC) (\%), as affected by genotype (G) and PEG concentration (C) after 10 and 15 days of PEG stress

\begin{tabular}{|c|c|c|c|c|c|c|c|c|}
\hline & & & & PEG Co & entration (9 & (C) & & \\
\hline & $\begin{array}{c}\text { Genotype } \\
\text { (G) }\end{array}$ & 0 & $\begin{array}{c}5 \\
(-0.05 \mathrm{MPa})\end{array}$ & $\begin{array}{c}10 \\
(-0.15 \mathrm{MPa})\end{array}$ & $\begin{array}{c}20 \\
(-0.5 \mathrm{MPa})\end{array}$ & $\begin{array}{c}30 \\
(-1 \mathrm{MPa})\end{array}$ & $\begin{array}{c}50 \\
(2.7 \mathrm{MPa})\end{array}$ & $\begin{array}{c}\% \\
\text { Mean } \\
\text { (C) }\end{array}$ \\
\hline & 'Gavdos' & 63.7 & 66.5 & 63.7 & 57.0 & 49.4 & 53.4 & $59 \mathrm{~b}$ \\
\hline & 'Keryneia' & 68.5 & 67.5 & 67.2 & 62.8 & 50.0 & 52.3 & $61.4 \mathrm{a}$ \\
\hline & 'Macarena' & 65.5 & 63.7 & 62.5 & 53.3 & 44.1 & 45.6 & $55.7 \mathrm{c}$ \\
\hline $10^{\text {th }} \mathrm{dav}$ & 'CAT16-31' & 67.7 & 66.2 & 66.5 & 60.5 & 47.7 & 51.8 & $60.1 \mathrm{ab}$ \\
\hline & 'CAT16-27’ & 60.7 & 55.7 & 46.2 & 42.8 & 45.4 & 47.9 & $49.7 \mathrm{e}$ \\
\hline & 'Thiva' & 59.7 & 60.5 & 55.8 & 59.7 & 46.5 & 46.4 & $54.9 \mathrm{~cd}$ \\
\hline & 'CAT16-4' & 62.2 & 63.0 & 56.5 & 50.0 & 48.3 & 46.8 & $54.5 \mathrm{~cd}$ \\
\hline & 'Sifnos' & 60.5 & 58.7 & 54.7 & 55.2 & 44.0 & 46.2 & $53.2 \mathrm{~d}$ \\
\hline & 'Line 9/14' & 65.0 & 60.0 & 56.5 & 51.8 & 45.0 & 44.4 & $53.8 \mathrm{~cd}$ \\
\hline & 'Lemnos' & 60.5 & 58.5 & 58.5 & 37.5 & 51.0 & 50.0 & $52.7 \mathrm{~d}$ \\
\hline & Mean $(G)$ & $63.4 \mathrm{a}$ & $62 \mathrm{a}$ & $58.8 \mathrm{~b}$ & $53 \mathrm{c}$ & $47.1 \mathrm{~d}$ & $48.5 \mathrm{~d}$ & \\
\hline & & & & & & & & $\begin{array}{l}\text { Mean } \\
(\mathrm{C})\end{array}$ \\
\hline & 'Gavdos' & 75.8 & 77.8 & 73.0 & 60.3 & 49.4 & 54.1 & $65 \mathrm{a}$ \\
\hline & 'Keryneia' & 71.8 & 71.0 & 69.5 & 71.0 & 46.9 & 54.9 & $64.1 \mathrm{a}$ \\
\hline & 'Macarena' & 73.3 & 70.8 & 68.5 & 60.0 & 43.2 & 45.9 & $60.3 \mathrm{bc}$ \\
\hline & 'CAT16-31' & 70.0 & 67.8 & 68.5 & 67.8 & 42.8 & 50.6 & $61.3 \mathrm{~b}$ \\
\hline & 'CAT16-27' & 63.5 & 59.3 & 58.5 & 39.0 & 43.9 & 49.3 & $52.2 \mathrm{~g}$ \\
\hline $15^{\text {th }}$ day & 'Thiva' & 65.3 & 67.3 & 55.5 & 64.5 & 46.3 & 48.2 & $57.8 \mathrm{de}$ \\
\hline & 'CAT16-4’ & 68.8 & 71.3 & 65.0 & 53.8 & 48.4 & 48.3 & $\begin{array}{l}59.2 \\
\text { bcd }\end{array}$ \\
\hline & 'Sifnos' & 64.8 & 61.8 & 60.3 & 57.8 & 43.5 & 46.3 & 55.7 ef \\
\hline & 'Line 9/14' & 70.8 & 65.3 & 61.8 & 55.3 & 44.3 & 55.3 & $58.8 \mathrm{~cd}$ \\
\hline & 'Lemnos' & 63.8 & 61.0 & 60.5 & 40.2 & 53.2 & 50.8 & $54.8 \mathrm{f}$ \\
\hline & $\operatorname{Mean}(\mathrm{G})$ & $68.8 \mathrm{a}$ & $67.3 \mathrm{a}$ & $64.1 \mathrm{~b}$ & $56.9 c$ & $46.2 \mathrm{e}$ & $50.4 \mathrm{~d}$ & \\
\hline
\end{tabular}

Note: At each time interval (days), means followed by the same letter, within each factor, are not significantly different according to $\operatorname{LSD}(\mathrm{p} \leq 0.05)$.

In relation to traits associated with post-germination growth of seedlings, significant differences were observed both at stress and at genotype level. Specifically, for root length, although no differences were noted in mean values between controls, 5\% PEG and 10\% PEG, a significant decrease in root length was recorded at 20\%, 30\% and 50\% PEG (Table 4). Across stress levels, 'Thiva' had the highest mean for root length, while landrace 'Lemnos' had the highest root length at 30\% PEG. At high stress level (50\% PEG) however, most genotypes were incapable of forming roots, with the exception of 'Gavdos', 'Keryneia' and 'Lemnos'. For shoot length, the highest mean values were recorded at 5\% PEG, followed by control and 10\% PEG treatment (Table 5). At higher stress levels however, a drastic decrease in shoot length was observed, while treatment with $30 \%$ and 50\% PEG was associated with inability to form shoots in all genotypes tested. With respect to shoot length, 'Keryneia' was the best performing cultivar as it presented the highest mean value at 20\% PEG. Based on these findings, 'Thiva' and 'Keryneia' had the highest SVI value across stress levels, followed by 'Gavdos', while 'CAT16-27' ranked in the bottom. According to SVI, genotypes were classified in four distinct classes: i) SVI $>$ 450: 'Thiva', 'Keryneia,' ii) SVI = 350-450: 'Gavdos', iii) SVI = 250-350: 'CAT16-31', 'Macarena', 'CAT164 ' and iv) SVI < 250: 'Sifnos', 'Lemnos', 'Line 9/14' and 'CAT16-27' (Table 6).

In relation to correlations among traits, germination percentage showed significant positive correlation with root length $\left(r=0.667^{*}\right)$, while WC was well correlated with shoot length $\left(r=0.915^{* *}\right)$. In contrast, no 
correlation was found between germination percentage and 100 seed weight (seed size) at any stress level applied (Table 7).

Table 3. Seed water absorbance (WU) (\%), as affected by genotype (G) and PEG concentration (C) after 3,5 and 7 days of PEG stress

\begin{tabular}{|c|c|c|c|c|c|c|c|c|}
\hline Time & \multirow{3}{*}{ Genotype (G) } & \multicolumn{7}{|c|}{ PEG Concentration $(\%)(\mathrm{C})$} \\
\hline \multirow{13}{*}{$3^{\text {rd }}$ day } & & 0 & & 10 & 20 & 30 & 50 & $\%$ \\
\hline & & 0 & $(-0.05 \mathrm{MPa})$ & $(-0.15 \mathrm{MPa})$ & $(-0.5 \mathrm{MPa})$ & $(-1 \mathrm{MPa})$ & $(-2.7 \mathrm{MPa})$ & Mean $(C)$ \\
\hline & 'Gavdos' & 54.0 & 55.8 & 46.3 & 47.8 & 42.5 & 48.0 & $48.9 \mathrm{bc}$ \\
\hline & 'Keryneia' & 49.7 & 50.3 & 52.3 & 51.7 & 40.8 & 35.5 & $46.7 \mathrm{de}$ \\
\hline & 'Macarena' & 48.7 & 53.8 & 46.2 & 45.0 & 41.2 & 40.0 & $45.8 \mathrm{e}$ \\
\hline & 'CAT16-31' & 57.3 & 57.3 & 61.0 & 54.0 & 48.9 & 48.0 & $54.4 \mathrm{a}$ \\
\hline & 'CAT16-27’ & 53.3 & 51.0 & 50.0 & 46.8 & 47.9 & 47.4 & $49.3 \mathrm{~b}$ \\
\hline & 'Thiva' & 49.5 & 46.2 & 49.5 & 52.8 & 42.7 & 42.3 & $47.2 \mathrm{cde}$ \\
\hline & 'CAT16-4' & 55.8 & 52.8 & 52.3 & 43.3 & 50.0 & 45.6 & $49.9 \mathrm{~b}$ \\
\hline & 'Sifnos' & 58.3 & 55.5 & 56.5 & 52.8 & 51.6 & 47.7 & $53.6 \mathrm{a}$ \\
\hline & 'Line 9/14' & 53.0 & 49.7 & 52.5 & 45.5 & 48.9 & 45.7 & $49.2 \mathrm{~b}$ \\
\hline & 'Lemnos' & 50.0 & 47.7 & 52.7 & 48.3 & 45.5 & 44.8 & $48.2 \mathrm{bcd}$ \\
\hline & $\operatorname{Mean}(\mathrm{G})$ & $53 \mathrm{a}$ & $52 \mathrm{a}$ & $51.9 a$ & $48.8 \mathrm{~b}$ & $46 c$ & $44.5 \mathrm{~d}$ & \\
\hline \multirow{12}{*}{$5^{\text {th }}$ day } & & & & & & & & $\operatorname{Mean}(\mathrm{C})$ \\
\hline & 'Gavdos' & 56.8 & 57.0 & 50.8 & 48.5 & 45.4 & 47.5 & $50.9 \mathrm{cde}$ \\
\hline & 'Keryneia' & 55.8 & 52.8 & 53.5 & 50.3 & 39.8 & 37.9 & $48.3 \mathrm{f}$ \\
\hline & 'Macarena' & 50.8 & 54.3 & 49.3 & 46.5 & 38.3 & 38.2 & $46.2 \mathrm{~g}$ \\
\hline & 'CAT16-31' & 57.8 & 61.2 & 59.5 & 53.7 & 50.6 & 51.8 & $55.7 \mathrm{~b}$ \\
\hline & ‘CAT16-27’ & 55.5 & 53.3 & 50.8 & 44.5 & 46.0 & 46.8 & $49.4 \mathrm{ef}$ \\
\hline & 'Thiva' & 53.5 & 51.0 & 51.3 & 54.8 & 45.0 & 46.3 & $50.3 \mathrm{de}$ \\
\hline & 'CAT16-4’ & 61.5 & 55.5 & 56.5 & 46.3 & 48.5 & 46.1 & $52.3 \mathrm{c}$ \\
\hline & 'Sifnos' & 63.3 & 60.3 & 58.5 & 59.5 & 53.4 & 52.6 & $57.8 \mathrm{a}$ \\
\hline & 'Line 9/14' & 56.5 & 55.5 & 54.5 & 51.0 & 48.0 & 45.6 & $51.9 \mathrm{~cd}$ \\
\hline & 'Lemnos' & 54.8 & 54.0 & 54.0 & 47.7 & 51.5 & 44.9 & $51.1 \mathrm{cde}$ \\
\hline & $\operatorname{Mean}(G)$ & $56.6 \mathrm{a}$ & $55.5 \mathrm{a}$ & $53.9 \mathrm{~b}$ & $50.3 c$ & $46.6 \mathrm{~d}$ & $45.8 \mathrm{~d}$ & \\
\hline \multirow{12}{*}{$7^{\text {th }}$ day } & & & & & & & & $\operatorname{Mean}(\mathrm{C})$ \\
\hline & 'Gavdos' & 56.3 & 58.5 & 50.5 & 46.5 & 47.0 & 47.6 & $51 \mathrm{~cd}$ \\
\hline & 'Keryneia' & 58.8 & 58.3 & 57.8 & 57.0 & 40.6 & 39.0 & $51.9 \mathrm{bc}$ \\
\hline & 'Macarena' & 56.5 & 57.3 & 52.0 & 48.0 & 41.0 & 40.0 & $49.2 \mathrm{~d}$ \\
\hline & 'CAT16-31' & 62.0 & 62.8 & 63.3 & 56.2 & 48.2 & 52.1 & $57.4 \mathrm{a}$ \\
\hline & 'CAT16-27’ & 57.8 & 53.0 & 51.0 & 45.0 & 45.5 & 48.5 & $50.1 \mathrm{~cd}$ \\
\hline & 'Thiva' & 52.5 & 52.5 & 52.8 & 53.8 & 41.2 & 43.9 & $49.4 \mathrm{~d}$ \\
\hline & 'CAT16-4’ & 62.8 & 57.5 & 60.5 & 49.3 & 45.7 & 45.0 & $53.4 \mathrm{~b}$ \\
\hline & 'Sifnos' & 63.5 & 61.3 & 61.3 & 60.7 & 49.8 & 51.9 & $58 \mathrm{a}$ \\
\hline & 'Line 9/14' & 58.3 & 61.0 & 56.5 & 52.0 & 47.7 & 44.5 & $53.3 \mathrm{~b}$ \\
\hline & 'Lemnos' & 53.3 & 52.0 & 51.7 & 43.2 & 47.7 & 46.1 & $48.9 \mathrm{~d}$ \\
\hline & Mean $(G)$ & $58.2 \mathrm{a}$ & $57.4 \mathrm{a}$ & $55.7 \mathrm{~b}$ & $51.2 \mathrm{c}$ & $45.4 \mathrm{~d}$ & $45.9 \mathrm{~d}$ & \\
\hline
\end{tabular}

Note: At each time interval (days), means followed by the same letter, within each factor, are not significantly different according to $\operatorname{LSD}(\mathrm{p} \leq 0.05)$. 
Koskosidis A et al. (2020). Not Bot Horti Agrobo 48(1):294-304

300

Table 4. Root length $(\mathrm{cm})$, as affected by genotype $(\mathrm{G})$ and PEG concentration $(\mathrm{C})$

\begin{tabular}{|c|c|c|c|c|c|c|c|c|}
\hline \multirow[b]{2}{*}{ Time } & \multirow[b]{2}{*}{ Genotype (G) } & \multicolumn{7}{|c|}{ PEG Concentration (\%) (C) } \\
\hline & & 0 & $\begin{array}{c}5 \\
(-0.05 \mathrm{MPa})\end{array}$ & $\begin{array}{c}10 \\
(-0.15 \mathrm{MPa})\end{array}$ & $\begin{array}{c}20 \\
(-0.5 \mathrm{MPa})\end{array}$ & $\begin{array}{c}30 \\
(-1 \mathrm{MPa})\end{array}$ & $\begin{array}{c}50 \\
(-2.7 \mathrm{MPa})\end{array}$ & $\begin{array}{l}\text { Mean } \\
(\mathrm{C})\end{array}$ \\
\hline \multirow{11}{*}{$15^{\text {th }}$ day } & 'Gavdos' & 2.900 & 3.664 & 3.735 & 3.167 & 0.680 & 0.592 & $2.456 \mathrm{bc}$ \\
\hline & 'Keryneia' & 4.377 & 3.201 & 4.060 & 4.600 & 0.752 & 0.585 & $2.929 \mathrm{~b}$ \\
\hline & 'Macarena' & 3.674 & 5.367 & 2.820 & 3.126 & 0.000 & 0.000 & $2.498 \mathrm{bc}$ \\
\hline & 'CAT16-31' & 2.987 & 3.237 & 3.615 & 2.958 & 0.030 & 0.000 & $2.138 \mathrm{c}$ \\
\hline & ‘CAT16-27’ & 2.695 & 1.500 & 1.385 & 0.000 & 0.935 & 0.000 & $1.086 \mathrm{~d}$ \\
\hline & 'Thiva' & 4.767 & 5.127 & 5.262 & 7.455 & 1.025 & 0.000 & $3.94 \mathrm{a}$ \\
\hline & 'CAT16-4’ & 3.487 & 4.558 & 3.035 & 1.327 & 1.015 & 0.000 & $2.237 \mathrm{c}$ \\
\hline & 'Sifnos' & 3.462 & 2.615 & 3.481 & 2.552 & 0.055 & 0.000 & $2.028 \mathrm{c}$ \\
\hline & 'Line 9/14' & 2.235 & 1.715 & 1.490 & 2.047 & 0.225 & 0.000 & $1.285 \mathrm{~d}$ \\
\hline & 'Lemnos' & 2.335 & 1.975 & 4.020 & 0.295 & 2.482 & 0.253 & $1.893 \mathrm{c}$ \\
\hline & Mean $(\mathrm{G})$ & $3.292 \mathrm{a}$ & $3.296 \mathrm{a}$ & $3.29 \mathrm{a}$ & $2.753 \mathrm{~b}$ & $0.72 \mathrm{c}$ & $0.143 \mathrm{~d}$ & \\
\hline
\end{tabular}

Note: At each time interval (days), means followed by the same letter, within each factor, are not significantly different according to $\operatorname{LSD}(\mathrm{p} \leq 0.05)$

Table 5. Shoot length (cm), as affected by genotype (G) and PEG concentration (C)

\begin{tabular}{|c|c|c|c|c|c|c|c|c|}
\hline \multirow[b]{2}{*}{ Time } & \multirow[b]{2}{*}{ Genotype $(G)$} & \multicolumn{7}{|c|}{ PEG Concentration (C) } \\
\hline & & 0 & $\begin{array}{c}5 \\
(-0.05 \mathrm{MPa})\end{array}$ & $\begin{array}{c}10 \\
(-0.15 \mathrm{MPa})\end{array}$ & $\begin{array}{c}20 \\
(-0.5 \mathrm{MPa})\end{array}$ & $\begin{array}{c}30 \\
(-1 \mathrm{MPa})\end{array}$ & $\begin{array}{c}50 \\
(-2.7 \mathrm{MPa})\end{array}$ & $\begin{array}{c}\text { Mean } \\
(\mathrm{C})\end{array}$ \\
\hline \multirow{11}{*}{$15^{\text {th }}$ day } & 'Gavdos' & 1.765 & 4.183 & 3.918 & 0.584 & 0.000 & 0.000 & $1.742 \mathrm{~b}$ \\
\hline & 'Keryneia' & 3.006 & 2.650 & 3.865 & 3.505 & 0.000 & 0.000 & $2.171 \mathrm{a}$ \\
\hline & 'Macarena' & 2.212 & 3.379 & 2.290 & 0.815 & 0.000 & 0.000 & $1.449 \mathrm{c}$ \\
\hline & 'CAT16-31' & 1.596 & 2.200 & 3.005 & 1.547 & 0.000 & 0.000 & $1.391 \mathrm{~cd}$ \\
\hline & 'CAT16-27’ & 0.680 & 0.458 & 0.225 & 0.000 & 0.000 & 0.000 & $0.227 \mathrm{e}$ \\
\hline & 'Thiva' & 1.405 & 2.188 & 1.145 & 2.145 & 0.000 & 0.000 & $1.147 \mathrm{~cd}$ \\
\hline & 'CAT16-4' & 2.153 & 3.248 & 1.185 & 0.035 & 0.000 & 0.000 & $1.103 \mathrm{~d}$ \\
\hline & 'Sifnos' & 0.895 & 0.438 & 0.560 & 0.530 & 0.000 & 0.000 & $0.404 \mathrm{e}$ \\
\hline & 'Line 9/14' & 1.033 & 1.093 & 0.600 & 0.200 & 0.000 & 0.000 & $0.487 \mathrm{e}$ \\
\hline & 'Lemnos' & 0.580 & 0.752 & 0.270 & 0.100 & 0.000 & 0.000 & $0.284 \mathrm{e}$ \\
\hline & Mean $(G)$ & $1.532 \mathrm{~b}$ & $2.059 \mathrm{a}$ & $1.706 \mathrm{~b}$ & $0.946 \mathrm{c}$ & $0.000 \mathrm{~d}$ & $0.000 \mathrm{~d}$ & \\
\hline
\end{tabular}

Note: At each time interval (days), means followed by the same letter, within each factor, are not significantly different according to LSD $(\mathrm{p} \leq 0.05)$.

Table 6. Seedling vigor index (SVI) as affected by genotype (G) and PEG concentration (C)

\begin{tabular}{|c|c|c|c|c|c|c|c|c|}
\hline \multirow[b]{2}{*}{ Time } & \multirow{2}{*}{$\begin{array}{c}\text { Genotype } \\
\text { (G) }\end{array}$} & \multicolumn{7}{|c|}{ PEG Concentration (C) } \\
\hline & & 0 & $\begin{array}{c}5 \\
(-0.05 \mathrm{MPa})\end{array}$ & $\begin{array}{c}10 \\
(-0.15 \mathrm{MPa})\end{array}$ & $\begin{array}{c}20 \\
(-0.5 \mathrm{MPa})\end{array}$ & $\begin{array}{c}30 \\
(-1 \mathrm{MPa})\end{array}$ & $\begin{array}{c}50 \\
(-2.7 \mathrm{MPa})\end{array}$ & $\begin{array}{l}\text { Mean } \\
(\mathrm{C})\end{array}$ \\
\hline \multirow{11}{*}{$15^{\text {th }}$ day } & 'Gavdos' & 441.0 & 762.0 & 749.5 & 276.3 & 45.0 & 4.5 & $379.7 \mathrm{~b}$ \\
\hline & 'Keryneia' & 702.0 & 537.5 & 792.5 & 779.3 & 23.0 & 7.0 & $473.5 \mathrm{a}$ \\
\hline & 'Macarena' & 569.0 & 818.8 & 451.8 & 151.0 & 0,0 & 0.0 & $331.8 \mathrm{bc}$ \\
\hline & 'CAT16-31' & 395.0 & 492.0 & 623.2 & 404.5 & 0,0 & 0.0 & $319.1 \mathrm{bc}$ \\
\hline & 'CAT16-27’ & 315.7 & 190.0 & 74.7 & 0,0 & 51.2 & 0.0 & $105.3 \mathrm{f}$ \\
\hline & 'Thiva' & 609.5 & 731.5 & 635.5 & 948.7 & 61.0 & 0.0 & $497.7 \mathrm{a}$ \\
\hline & 'CAT16-4' & 549.0 & 763.7 & 397.0 & 23.5 & 57.0 & 0.0 & $298.4 \mathrm{c}$ \\
\hline & 'Sifnos' & 424.5 & 288.8 & 355.7 & 262.0 & 0.0 & 0.0 & $221.8 \mathrm{~d}$ \\
\hline & 'Line 9/14' & 301.7 & 246.8 & 185.2 & 153.2 & 10.3 & 0.0 & $149.5 \mathrm{ef}$ \\
\hline & 'Lemnos' & 280.0 & 238.5 & 333.8 & 30.7 & 201.5 & 1.5 & $181 \mathrm{de}$ \\
\hline & Mean $(G)$ & $458.7 \mathrm{~b}$ & $507 a$ & $459.9 \mathrm{~b}$ & $302,9 \mathrm{c}$ & $44.9 \mathrm{~d}$ & $1.3 \mathrm{e}$ & \\
\hline
\end{tabular}

Note: At each time interval (days), means followed by the same letter, within each factor, are not significantly different according to $\operatorname{LSD}(\mathrm{p} \leq 0.05)$. 
Table 7. Correlations between germination percentage and traits related to germination and early seedling growth

\begin{tabular}{|c|c|c|c|c|c|c|c|}
\hline \multicolumn{2}{|c|}{ Traits and correlation } & \multirow{2}{*}{$\begin{array}{c}\text { Germination } \\
1\end{array}$} & \multirow{2}{*}{$\begin{array}{c}\begin{array}{c}\text { Seed } \\
\text { weight }\end{array} \\
-0.295\end{array}$} & \multirow{2}{*}{$\begin{array}{c}\text { WU } \\
-0.095\end{array}$} & \multirow{2}{*}{$\begin{array}{c}\text { WC } \\
0.386\end{array}$} & \multirow{2}{*}{$\begin{array}{c}\begin{array}{c}\text { Shoot } \\
\text { length }\end{array} \\
0.359\end{array}$} & \multirow{2}{*}{$\begin{array}{r}\begin{array}{r}\text { Root } \\
\text { length }\end{array} \\
0.667^{*}\end{array}$} \\
\hline \multirow{3}{*}{ Germination } & Pearson Correlation & & & & & & \\
\hline & Sig. (2-tailed) & & 0.408 & 0.795 & 0.271 & 0.308 & 0.035 \\
\hline & $\mathrm{N}$ & 10 & 10 & 10 & 10 & 10 & 10 \\
\hline \multirow{3}{*}{ Seed weight } & Pearson Correlation & -.295 & 1 & -0.073 & 0.225 & 0.190 & -0.001 \\
\hline & Sig. (2-tailed) & .408 & & 0.842 & 0.532 & 0.598 & 0.998 \\
\hline & $\mathrm{N}$ & 10 & 10 & 10 & 10 & 10 & 10 \\
\hline \multirow{3}{*}{ WU } & Pearson Correlation & -0.095 & -0.073 & 1 & 0.099 & -0.046 & -0.220 \\
\hline & Sig. (2-tailed) & 0.795 & 0.842 & & 0.785 & 0.900 & 0.542 \\
\hline & $\mathrm{N}$ & 10 & 10 & 10 & 10 & 10 & 10 \\
\hline \multirow{3}{*}{ WC } & Pearson Correlation & 0.386 & 0.225 & 0.099 & 1 & $0.915^{* *}$ & 0.447 \\
\hline & Sig. (2-tailed) & 0.271 & 0.532 & 0.785 & & 0.000 & 0.196 \\
\hline & $\mathrm{N}$ & 10 & 10 & 10 & 10 & 10 & 10 \\
\hline \multirow{3}{*}{ Shoot length } & Pearson Correlation & 0.359 & 0.190 & -0.046 & $0.915^{* *}$ & 1 & 0.626 \\
\hline & Sig. (2-tailed) & 0.308 & 0.598 & 0.900 & 0.000 & & 0.053 \\
\hline & $\mathrm{N}$ & 10 & 10 & 10 & 10 & 10 & 10 \\
\hline \multirow{3}{*}{ Root length } & Pearson Correlation & $0.667^{*}$ & -0.001 & -0.220 & 0.447 & 0.626 & 1 \\
\hline & Sig. (2-tailed) & 0.035 & 0.998 & 0.542 & 0.196 & 0.053 & \\
\hline & $\mathrm{N}$ & 10 & 10 & 10 & 10 & 10 & 10 \\
\hline
\end{tabular}

${ }^{*}$ Correlation is significant at the 0.05 level (2-tailed).

** Correlation is significant at the 0.01 level (2-tailed).

\section{Discussion}

Drought is a major abiotic factor limiting plant growth, development and productivity in vast majority of crop species worldwide. Nearly one third of cultivated areas is subjected to water scarcity, while according to recent estimates, based on climatic projections, the unavailability of water resources is expected to intensify (Ceccareli et al., 2010). Considering the obvious necessity to focus on breeding for drought tolerance, the identification of traits related to drought tolerance and water uptake (Valladares et al., 2004) is becoming increasingly important. Germination as being one of the most critical stages, due to its correlation with seedling establishment and early growth (Kaydan and Yagmur, 2008), has been proposed as a suitable criterion for screening drought tolerance. In this framework, this study was aimed at determining the response of ten chickpea genotypes to PEG-induced drought stress at germination phase as an approach to select for drought tolerant genotypes.

As far as germination potential is concerned, our results are in accordance to those of relevant studies and provide further evidence that drought significantly affects germination, with the effects of stress being more severe as the intensity of stress increases (Jamaati-e-Somarin and Zabihi-e-Mahmoodabad, 2011; Wu et al., 2011; Haouari et al., 2013; Foti et al., 2018). For chickpea, it has been proposed that the threshold osmotic potential is $-0.8 \mathrm{MPa}$ (Yucel et al., 2010), while other studies report even lower threshold levels (Kalefetoglu et al., 2009). Contrasting such reports, $70 \%$ of genotypes employed in this study were able to germinate at -1 $\mathrm{MPa}$, thus surpassing the osmotic potential threshold of -0.8 MPa. Further, a portion of them, namely 'Gavdos', 'Keryneia' and 'Lemnos', was capable of germinating, yet at low rates, under severe osmotic stress conditions (2.7 $\mathrm{MPa}$ ). It is worth noting however, that genotypes presented significant variations in relation to germination 
potential at different stress levels applied. In this line, 'Thiva' ranked at the top across treatments while, at the same time, exhibiting a high germination rate (>99\%) at 20\% PEG, followed by a drastic decline at $30 \%$ PEG and total repression of germination (0\%) at 50\% PEG. On the other hand, landrace 'Lemnos' exhibited a high germination rate $(79 \%)$ at $30 \%$ PEG, which was pinpointed as the critical stress level where germination rate is most profoundly decreased, therefore providing evidence for its drought tolerance as well as for its possible exploitation as valuable genetic material for breeding activities.

Given that water availability affects germination potential (Hodge et al., 2009), via regulating the synthesis of hydrolytic enzymes involved in processes of seedling tissue synthesis and radicle elongation (Canas et al., 2006), WC and WU are directly affected by drought stress (Muscolo et al., 2014). Among genotypes with high germination rate, 'Keryneia' and 'Gavdos' presented the highest values for WC and WU, thus indicating a more efficient water uptake. However, landrace 'Lemnos' followed by 'Thiva', despite their high germination rates, ranked very low at WC and WU, therefore suggesting a different varietal pattern in terms of water demands to initiate the germination process.

In relation to traits related to post-germination, and in particular root and shoot length, their response differs substantially under drought stress, with shoot tissues being more susceptible to water deficiency (Okcu et al., 2005; Abd Allah et al., 2010). In our study, at 30\% PEG-induced stress, all genotypes were incapable of shoot formation, whereas under the same stress conditions they managed to develop roots. Further, germination percentage showed positive correlation only with root length $\left(r=0.67^{*}\right)$. Such findings are indicative of the fact that shoot length was more affected than root length and are in agreement with previous studies suggesting that the decreased osmotic potential leads to a more drastic inhibition of shoot tissue elongation (Kalefetoglu et al., 2009; Yucel et al., 2010). It is interesting that the majority of genotypes showed high length either for roots or shoots, with the exception of cultivars 'Thiva' and 'Keryneia' which exhibited high values both for shoot and root length. Consequently, 'Thiva' and 'Keryneia' ranked at the top in relation to SVI, followed by 'Gavdos'. In contrast, landrace 'Lemnos', which showed an increased germination potential, presented low SVI due to its low shoot and root elongation rate. Based on SVI, genotypes were classified in four categories: cultivars 'Thiva' and 'Keryneia' were characterised as drought tolerant (SVI > 450), 'Gavdos' as moderately tolerant (SVI: 350-450), 'CAT16-31', 'Macarena' and 'CAT16-4' as moderately susceptible (SVI: 250-350) and 'Sifnos', 'Lemnos', 'Line 9/14' and 'CAT16-27' as susceptible (SVI < 250).

In total, findings of this study underline that the determination of genotypic response to drought stress may be readily pursued at germination phase in chickpea and further indicate that the classification of genotypes in terms of drought tolerance may be performed on the basis of traits associated to seed germination and post-germination growth of seedlings. Given that previous studies have proven a good correlation between in vitro and field germination potential under drought stress in wheat, providing similar ranking for genotypic performance in in vitro and field conditions (Khakwani et al., 2011), such findings are of outmost importance. Upon verification of the fact that the deduced classification of genotypes is well correlated to their field performance under drought conditions, this approach may be exploited for selecting drought tolerant chickpea genotypes at early growth stages.

\section{Conclusions}

Overall findings revealed the existence of significant genetic variability in relation to drought tolerance among chickpea genotypes evaluated. Further, the results underline the fact that SVI may be employed as an appropriate criterion for screening drought tolerance in chickpea. To this respect, cultivars 'Thiva' and 'Keryneia', followed by 'Gavdos', exhibited superior performance in terms of drought tolerance, thus suggesting their possible use for cultivation in dry areas. The aforementioned varieties, along with landrace 'Lemnos', may further used in pure line selection or hybridization breeding programs targeted at the development of varieties suitable for areas where water scarcity is a major constraint. 


\section{Acknowledgements}

This research received no specific grant from any funding agency in the public, commercial, or not-forprofit sectors. The authors wish to thank Dr. Dimitra Loka for her valuable comments on the manuscript.

\section{Conflict of Interests}

The authors declare that there are no conflicts of interest related to this article.

\section{References}

Abd Allah AA, Badawy Shimaa A, Zayed BA, ElGohary AA (2010). The role of root system traits in the drought tolerance of rice (Oryza sativa L.). World Academy of Science, Engineering and Technology 68:378-1382.

Abdual-Baki AA, Anderson JD (1973). Relationship between decarboxilation of glutamic acid and vigour in soybean seed. Crop Science 13:222-226.

Arjenaki FG, Dehaghi MA, Jabbari R (2011). Effects of priming on seed germination of Marigold (Calendula officinalis). Advance in Environmental Biology 5:276-280.

Arnoldi A, Zanoni C, Lamm, C, Boschin G, (2015). The role of grain legumes in the prevention of hypercholesterolemia and hypertension. Critical Reviews in Plant Sciences 34:144-168.

Black M, Pritchard H (2002). Desiccation and survival in plants drying without dying. New York, CABI publishing, pp 93-110.

Chaffei Haouari C, Hajjaji Nasraoui A, Carrayol E, Gouia H (2013). Variations in -, -amylase and -glycosidase activities in two genotypes of wheat under $\mathrm{NaCl}$ salinity stress. African Journal of Agricultural Research 8(18):2038-2043.

Canas RA, Canovas FM, Canton FR (2006). High levels of asparagines synthetase in hypocotyls of pine seedlings suggest a role of the enzyme in re-allocation of seed scord nitrogen. Planta 224:83-95.

Ceccarelli S, Grando S, Maatougui M, Michael M, Slash M, Haghparast R, ... Nachit M (2010). Climate change and agriculture plant breeding and climate changes. Journal of Agricultural Science 148:627-637.

Duc G, Agrama H, Bao S, Berger J, Bourion V, De Ron AM (2014). Breeding annual grain legumes for sustainable agriculture: new methods to approach complex traits and target new cultivar ideotypes. Critical Reviews in Plant Science 34:381411.

Foti C, Khah EM, Pavli OI (2018). Germination profiling of lentil genotypes subjected to salinity stress. Plant Biology 6(4):7583.

Hodge A, Berta G, Doussan C, Merchan F, Crespi M (2009). Plant root growth, architecture and function. Plant Soil 321:153187.

Jamaati-e-Somarin S, Zabihi-e-Mahmoodabad R (2011). Evaluation of drought tolerance indices of lentil varieties. Advances in Environmental Biology 5:581-584.

Jumrani K, Bhatia VS (2014). Impact of elevated temperatures on growth and yield of chickpea (Cicer arietinum L.). Field Crops Research 164:90-97.

Kalefetoglu Macar T, Turan O, Ekmekci Y (2009). Effect of water deficit induced by PEG and NaCl on chickpea (Cicer arientinum L.) cultivar and lines at early seedling stage. G.U. Journal of Science 22(1):5-14.

Kaydan D, Yagmur M (2008). Germination, seedling growth and relative water content of shoot in different seed sizes of triticale under osmotic stress of water and $\mathrm{NaCl}$. African Journal of Biotechnology 7:2862-2868.

Khakwani AA, Dennett M, Munir M (2011). Drought tolerance screening of wheat varieties by inducing water stress conditions. Songklanakarin Journal of Science and Technology 33:135-142.

Kulkarni M, Deshpande U (2007). In vitro screening of tomato genotypes for drought resistance using polyethylene glycol. African Journal of Biotechnology 6:691-696.

Michell BE, Kaufmann MR (1972). The osmotic potential of polyethylene glycol 6000. Plant Physiology 51:914-916. 
304

Mujeeb-ur-Rahman, Umed AS, Mohammad Z, Shereen G (2008). Effects of $\mathrm{NaCl}$ salinity on 5 wheat (Triticum aestivum L.) cultivars. World Journal of Agricultural Sciences 4(3):398-403.

Muscolo A, Sidari M, Anastasi U, Santonoceto C, Maggio A (2014). Effect of PEG-induced drought stress on seed germination of four lentil genotypes. Journal of Plant Interactions 9(1):354-363.

Okcu G, Kaya MD, Atak M (2005). Effects of salt and drought stresses on germination and seedling growth of pea (Pisum Sativum L.). Turkish Journal Agriculture and Forestry 29:237-242.

Rai K, Kalia RK, Singh R, Gangola P, Dhawan A (2011). Developing stress tolerant plants through in vitro selection an overview of the recent progress. Environmental and Experimental Botany 71:89-98.

Richards RA (1978). Variation between and within species of rapeseed (Brassica campestris and B. napus) in response to drought stress. III. Physiological and physicochemical characters. Australian Journal of Agriculture 29:491-501.

Rubiales D, Mikic A (2015.) Introduction: legumes in sustainable agriculture. Critical Reviews in Plant Science 34:2-3.

Sabaghpour SH (2003). Mechanism of drought tolerance in crops. Agricultural Aridity and Drought Scientific and Extension Quarterly of Jahad Agriculture 10:21-32.

Shaheen R, Hood-Nowotny RC (2005). Effect of drought and salinity on carbon isotope discrimination in wheat cultivars. Plant Science 168:901-909.

Siahsar BA, Ganjali S, Allahdoo M (2010). Evaluation of drought tolerance indices and their relationship with grain yield of lentil lines in drought-stressed and irrigated environments. Australian Journal of Basic Applied Science 4:4336-4346.

Siddique KHM, Regan KL, Tennant G, Thomson BD (2001). Water use and water use efficiency of cool season grain legumes in low rainfall Mediterranean-type environments. European Journal of Agronomy 15:267-280.

Valladares F, Vilagrosa A, Penuelas J, Ogaya R, Camarero JJ, Corchera L, Siso S, Gil-Pelegrin E (2004). Estres hidrico: ecofisiologia y escalas de la sequia [Water stress: ecophysiology and scales of drought]. In: Ecologia del Bosque Mediterraneo en un Mundo Cambiante, Madrid pp 163-190.

Venn BJ, Mann JI (2004). Cereal grains, legumes and diabetes. European Journal of Clinical Nutrition 58:1443-1461.

Wu C, Wang Q, Xie B, Wang Z, Cui J, Hu T (2011). Effects of drought and salt stress on seed germination of three leguminous species. African Journal of Biotechnology 10(78):17954-17961.

Yucel DO, Anlarsal AE, Mart D and Yucel C (2010). Effect of drought stress on early seedling growth of chickpea (Cicer arientinum L.) genotypes. World Applied Science Journal 11(4):478-485.

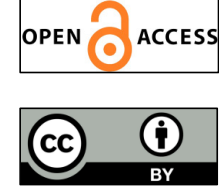

The journal offers free, immediate, and unrestricted access to peer-reviewed research and scholarly work. Users are allowed to read, download, copy, distribute, print, search, or link to the full texts of the articles, or use them for any other lawful purpose, without asking prior permission from the publisher or the author.

License - Articles published in Notulae Botanicae Horti Agrobotanici Cluj-Napoca are Open-Access, distributed under the terms and conditions of the Creative Commons Attribution (CC BY 4.0) License. (c) Articles by the authors; UASVM, Cluj-Napoca, Romania. The journal allows the author(s) to hold the copyright/to retain publishing rights without restriction. 